\title{
Monochromatic Matchings in the Shadow Graph of Almost Complete Hypergraphs
}

\author{
András Gyárfás $^{1 *}$, Gábor N. Sárközy ${ }^{1,2 \dagger}{ }^{\dagger}$, and Endre Szemerédi ${ }^{3}$ \\ ${ }^{1}$ Computer and Automation Research Institute, Hungarian Academy of Sciences, Budapest, \\ P.O. Box 63, Budapest H-1518, Hungary \\ gyarfas@sztaki.hu \\ ${ }^{2}$ Computer Science Department, Worcester Polytechnic Institute, Worcester, MA 01609, USA \\ gsarkozy@cs.wpi.edu \\ ${ }^{3}$ Department of Computer Science, Rutgers University, New Brunswick, NJ 08903, USA \\ szemered@cs.rutgers.edu
}

Received August 2, 2007

AMS Subject Classification: 05C15, 05C55, 05C65

\begin{abstract}
Edge colorings of $r$-uniform hypergraphs naturally define a multicoloring on the 2 -shadow, i.e., on the pairs that are covered by hyperedges. We show that in any $(r-1)$ coloring of the edges of an $r$-uniform hypergraph with $n$ vertices and at least $(1-\varepsilon)\left(\begin{array}{l}n \\ r\end{array}\right)$ edges, the 2-shadow has a monochromatic matching covering all but at most $o(n)$ vertices. This result confirms an earlier conjecture and implies that for any fixed $r$ and sufficiently large $n$, there is a monochromatic Berge-cycle of length $(1-o(1)) n$ in every $(r-1)$-coloring of the edges of $K_{n}^{(r)}$, the complete $r$-uniform hypergraph on $n$ vertices.
\end{abstract}

Keywords: colored complete uniform hypergraphs, monochromatic matchings

\section{Introduction}

Let $\mathcal{H}$ be an $r$-uniform hypergraph (a family of some $r$-element subsets of a set). The shadow graph of $\mathcal{H}$ is defined as the graph $\Gamma(\mathcal{H})$ on the same vertex set, where two vertices are adjacent if they are covered by at least one edge of $\mathcal{H}$. A coloring of the edges of an $r$-uniform hypergraph $\mathcal{H}, r \geq 2$, induces a multicoloring on the edges of the shadow graph $\Gamma(\mathcal{H})$ in a natural way; every edge $e$ of $\Gamma(\mathcal{H})$ receives the color of all hyperedges containing $e$. A subgraph of $\Gamma(\mathcal{H})$ is monochromatic if the color sets of its edges have a nonempty intersection.

A set of pairwise disjoint edges of the shadow graph covering $n-o(n)$ vertices is called an almost perfect matching of $\Gamma(\mathcal{H})$. Let $K_{n}^{(r)}$ denote the complete $r$-uniform

\footnotetext{
* Research supported in part by OTKA Grant No. K68322.

$\dagger$ Research supported in part by OTKA Grant No. K68322 and by a Janos Bolyai Research Scholarship.
} 
hypergraph on $n$ vertices. An $r$-uniform hypergraph is almost complete, if it has at least $(1-o(1))\left(\begin{array}{l}n \\ r\end{array}\right)$ edges. We call an $r$-uniform hypergraph $(1-\varepsilon)$-complete if it has at least $(1-\varepsilon) n^{r} / r$ ! edges.

In this paper we prove the following conjecture from [3].

Theorem 1.1. Assume that $r \geq 2$ is fixed, $\mathcal{H}$ is an almost complete r-uniform hypergraph with $n$ vertices, and its edges are colored with $r-1$ colors. Then the induced multicoloring on $\Gamma(\mathcal{H})$ contains a monochromatic almost perfect matching.

It is worth noting that Theorem 1.1 does not hold if we color with $r$ colors instead of $r-1$. An example in [3] gives an $r$-coloring of $K_{n}^{(r)}$ such that the largest number of vertices covered by any monochromatic matching is not larger than $\frac{(2 r-2) n}{2 r-1}$. In fact that is conjectured to be the best result ([3]) and proved for $r=3$ ([5]).

It was proved in [3] that Theorem 1.1 implies a stronger result, namely, that the almost perfect monochromatic matching $M$ guaranteed can be connected as well which means that the edges of $M$ are in the same component of the hypergraph defined by the edges of the color of $M$. Moreover, it was shown in [3] how to combine this strengthening of Theorem 1.1 and a "weak version" of the hypergraph Regularity lemma to get a Ramsey-type result for Berge-cycles. An $r$-uniform Berge-cycle ([1]) of length $\ell$ is a sequence of distinct vertices $v_{1}, v_{2}, \ldots, v_{\ell}$ together with a set of distinct edges $e_{1}, \ldots, e_{\ell}$ such that $e_{i}$ contains $v_{i}, v_{i+1}\left(v_{\ell+1} \equiv v_{1}\right)$.

Corollary 1.2. In every $(r-1)$-coloring of the edges of $K_{n}^{(r)}$ there is a monochromatic Berge-cycle of length at least $(1-o(1)) n$.

Note that in [3] it was conjectured that for sufficiently large $n$ this statement is true with a monochromatic Berge-cycle of length $n$, i.e., there is a monochromatic Hamiltonian Berge-cycle. However, at the moment we are unable to prove this stronger statement.

The way to obtain the above corollary from Theorem 1.1 illustrates a principle due to Łuczak (suggested in [8]): In many cases the task of finding a monochromatic path or cycle can be reduced to the easier task of finding a monochromatic matching via the Regularity lemma. This principle is applied in many recent Ramsey-type results such as [2-7].

\section{Proof of Theorem 1.1}

Suppose that $\mathcal{H}$ is a hypergraph with vertex set $V,|V|=n$ and $0<\delta<1$ is fixed.

Informally, a sequence $L$ of $k$ distinct vertices of $V$ is a $\delta$-bounded selection with respect to a family $\mathcal{F}$ of forbidden sets, if its elements are chosen in $k$ consecutive steps so that in each step there is a set $F \in \mathcal{F},|F| \leq \delta$ n such that vertices from $F$ cannot be included as the next element of the sequence.

Formally, to capture that the forbidden set $F$ for the next vertex of the sequence depends on the choices of previous vertices, we assume that the sets of $\mathcal{F}$ are indexed with certain sequences of vertices, called valid sequences. This is achieved by the following assumptions. The empty sequence $\emptyset$ is valid and there is a set $F(\emptyset) \in \mathcal{F}$. A one-element sequence $x_{1} \in V$ is valid if and only if $x_{1} \notin F(\emptyset)$. For $1 \leq i<k$ 
and for each valid sequence $\left(x_{1}, x_{2}, \ldots, x_{i}\right)$ of distinct vertices of $V$ there is a set $F\left(x_{1}, x_{2}, \ldots, x_{i}\right) \in \mathcal{F}$ and furthermore a sequence $\left(x_{1}, x_{2}, \ldots, x_{i}, x_{i+1}\right)$ is valid if and only if $x_{i+1} \notin F\left(x_{1}, x_{2}, \ldots, x_{i}\right)$.

Now, with the assumptions of the previous paragraph, a sequence $L=\left(x_{1}, x_{2}, \ldots\right.$, $x_{k}$ ) of $k$ distinct vertices is called a $\delta$-bounded selection with respect to $\mathcal{F}$ if $L$ is a valid sequence and each set in $\mathcal{F}$ has at most $\delta n$ vertices. As a trivial example, every sequence is $\delta$-bounded with respect to $\mathcal{F}$ that contains only empty sets. For the convenience of the reader a slightly less trivial example is described in the next paragraph.

Let $\mathcal{H}$ be a balanced complete tripartite graph on $n$ vertices and let $A(x)$ denote the partite class containing $x \in V$. Then define $\mathcal{F}$ by $F(\emptyset)=\emptyset$ and $F\left(x_{1}, x_{2}, \ldots, x_{i}\right)=$ $\cup_{j=1}^{i} A\left(x_{j}\right)$. With respect to these forbidden sets every $\frac{1}{3}$-bounded selection $L=$ $\left(x_{1}, x_{2}\right)$ is an edge in $\mathcal{H}$; every $\frac{2}{3}$-bounded selection $L=\left(x_{1}, x_{2}, x_{3}\right)$ is a triangle in $\mathcal{H}$. In fact, no matter how forbidden sets are defined, the numbers $\frac{1}{3}, \frac{2}{3}$ can not be lowered without violating the statements above, that is, for a $\delta<\frac{1}{3}$ not every $\delta$ bounded selection $L=\left(x_{1}, x_{2}\right)$ will be an edge in $\mathcal{H}$, and similarly, for a $\delta^{\prime}<\frac{2}{3}$ not every $\delta^{\prime}$-bounded selection $L=\left(x_{1}, x_{2}, x_{3}\right)$ will be a triangle in $\mathcal{H}$.

The following lemma from [3] ensures that in almost complete hypergraphs there exists $\mathcal{F}$, such that with respect to $\mathcal{F}$ every $\delta$-bounded selection of at most $r$ vertices is contained in many edges of the hypergraph.

Lemma 2.1. Assume that $\varepsilon>0, \delta=\varepsilon^{2^{-r}}$ and $\mathcal{H}$ is a $(1-\varepsilon)$-complete r-uniform hypergraph on $n$ vertices. Then there exists $\mathcal{F}$, such that with respect to $\mathcal{F}$ there exist $\delta$-bounded selections and every $\delta$-bounded selection $L \subset V(\mathcal{H})$ of at most $r$ vertices has the following property: L is contained in at least $(1-\delta) \frac{n^{r-|L|}}{(r-|L|) !}$ edges of $\mathcal{H}$. In particular, for $|L|=r, L$ is an edge of $\mathcal{H}$.

Now we are ready to prove Theorem 1.1 by induction on $r$. Let $\varepsilon>0$ be arbitrary, $\mathcal{H}$ is a $(1-\varepsilon)$-complete $r$-uniform hypergraph with $n$ vertices whose edges are colored with $r-1$ colors. We shall prove that there is a monochromatic matching $M$ in $\Gamma(\mathcal{H})$ covering all but at most $\alpha n$ vertices, where $\alpha$ tends to 0 if $\varepsilon$ tends to 0 .

Set $p=\sqrt{\delta} n+1$. For $r=2$ we have a $(1-\varepsilon)$-complete graph (colored with one color). Select a maximum matching $M$ in the graph. Observe that the set of vertices uncovered by $M$ forms an independent set. Since $\left(\begin{array}{l}p \\ 2\end{array}\right)>\frac{\delta n^{2}}{2}>\frac{\varepsilon n^{2}}{2}$, less than $p=o(n)$ vertices are uncovered by $M$.

Assume that Theorem 1.1 is true for every $q<r$. Consider an $(r-1)$-coloring of a $(1-\varepsilon)$-complete $r$-uniform hypergraph $\mathcal{H}$ with $r \geq 3$. Set $\delta=\varepsilon^{2^{-r}}$ and apply Lemma 2.1 to define $\mathcal{F}$. Consider all $\delta$-bounded selections of $r$ vertices with respect to $\mathcal{F}$. Let $G$ be the graph defined on the set of vertices that appear as the first vertices of these $\delta$-bounded selections where the edges are those edges of the shadow graph $\Gamma(\mathcal{H})$ that appear as the first two vertices of these $\delta$-bounded selections. Note that $G$ has minimum degree at least $(1-2 \delta) n$. Indeed, if $x_{1} \in V(G)$ then at most $\delta n$ vertices are forbidden for $x_{2}$ and another at most $\delta$ vertices are not in $V(G)$. On the other hand, for the remaining $(1-2 \delta) n$ vertices Lemma 2.1 (with $|L|=2$ ) ensures that $\left\{x_{1}, x_{2}\right\}$ is covered by at least $(1-\delta) \frac{n^{r-2}}{(r-2) !}>0$ edges of $\mathcal{H}$, thus $x_{1} x_{2} \in E(G)$. 
For any $v \in V(G)$ and $1 \leq i \leq r-1$, let $A_{i}$ be the set of vertices $w$ such that color $i$ is not on the edge $v w \in E(G)$, without loss of generality, $\left|A_{1}\right| \leq\left|A_{2}\right| \leq \cdots \leq\left|A_{r-1}\right|$. Assume that $y_{i} \in A_{i}$ for $1 \leq i \leq r-1$ are distinct vertices. Then $e=\left\{v, y_{1}, \ldots, y_{r-1}\right\} \notin$ $E(\mathcal{H})$ because no color can be assigned to the $r$-tuple $e$. Therefore, with

$$
D=\frac{\left|A_{1}\right|\left(\left|A_{2}\right|-1\right)\left(\left|A_{3}\right|-2\right) \cdots\left(\left|A_{r-1}\right|-r+2\right)}{(r-1) !},
$$

$v$ is contained in at least $D r$-tuples of $V(\mathcal{H})$ that are not edges of $\mathcal{H}$. However, Lemma 2.1 (with $|L|=1$ ) guarantees that the number of these $r$-tuples is less than

$$
\left(\begin{array}{c}
n-1 \\
r-1
\end{array}\right)-(1-\delta) \frac{n^{r-1}}{(r-1) !}<\frac{\delta n^{r-1}}{(r-1) !}
$$

Now it follows that

$$
\frac{\left(\left|A_{1}\right|-r+2\right)^{r-1}}{(r-1) !}<D<\frac{\delta n^{r-1}}{(r-1) !},
$$

implying that $\left|A_{1}\right|<n \delta^{\frac{1}{r-1}}+r-2$. Using this and the minimum degree condition in $G, v$ is adjacent in color 1 to at least $(1-2 \delta-\rho) n$ vertices of $G$, where $\rho=$ $\delta^{\frac{1}{(r-1)}}+\frac{r-2}{n}$.

The argument above can be repeated for any $v \in V(G)$, showing that $V(G)=$ $\cup_{i=1}^{r-1} X_{i}$ where $v \in X_{i}$ has the property that at least $(1-2 \delta-\rho) n$ edges of $G$ of color $i$ are incident to $v$.

Let $M_{i}$ be a maximum matching in color $i$ in the subgraph of $G$ induced by $V(G) \backslash$ $X_{i}$ and set $Y_{i}=V(G) \backslash\left(V\left(M_{i}\right) \cup X_{i}\right)$. Observe that, from the choice of $M_{i}$, no edge of $G$ within $Y_{i}$ is colored with color $i$.

If

$$
p(i-1)+\frac{\left|X_{i}\right|}{1-\delta} \geq\left|Y_{i}\right|
$$

holds for some $i, 1 \leq i \leq r-1$, then we have the required large matching in color $i$. Indeed, almost every edge of $G$ incident to $X_{i}$ has color $i$ thus $M_{i}$ can be extended to a matching that misses at most $p(i-1)+(3 \delta+\rho) n=o(n)$ vertices of $G$.

Assume that $p(i-1)+\frac{\left|X_{i}\right|}{1-\delta}<\left|Y_{i}\right|$ for every $i, 1 \leq i \leq r-1$. This implies that

$$
\begin{aligned}
\sum_{i=1}^{r-1}\left|Y_{i}\right| & >p \sum_{i=1}^{r-1}(i-1)+\frac{1}{1-\delta} \sum_{i=1}^{r-1}\left|X_{i}\right| \\
& \geq p\left(\begin{array}{c}
r-1 \\
2
\end{array}\right)+\frac{|V(G)|}{1-\delta} \\
& \geq p\left(\begin{array}{c}
r-1 \\
2
\end{array}\right)+\frac{(1-\delta) n}{1-\delta} \\
& =p\left(\begin{array}{c}
r-1 \\
2
\end{array}\right)+n .
\end{aligned}
$$


We claim that this inequality implies $\left|Y_{i} \cap Y_{j}\right| \geq p$ for some $1 \leq i<j \leq r-1$. Indeed, otherwise

$$
n \geq\left|\cup_{i=1}^{r-1} Y_{i}\right|>\sum_{i=1}^{r-1}\left|Y_{i}\right|-\left(\begin{array}{c}
r-1 \\
2
\end{array}\right) p,
$$

contradicting to the inequality above. This proves the claim.

Select $Y_{i}, Y_{j}$ from the claim, without loss of generality, $\left|Y_{r-2} \cap Y_{r-1}\right| \geq p$. From the definition of $p,\left(\begin{array}{l}p \\ 2\end{array}\right)>\frac{\delta n^{2}}{2}$ follows, implying from the minimum degree condition in $G$ that there are $x, y \in Y_{r-2} \cap Y_{r-1}$ such that $x y \in E(G)$ - this implies that the colors $r-2, r-1$ are not among the colors of $x y$. Notice that for $r=3$ we have a contradiction on this branch of the proof, $x y \in E(G)$ thus $\{x, y\}$ is covered by an edge of $\mathcal{H}$ (a triple) but it cannot have a color. Thus we may assume that $r \geq 4$.

Consider the $(r-2)$-uniform colored hypergraph $\mathcal{H}^{*}$ with edge set $\{e \backslash\{x, y\}: e \in$ $\mathcal{H}$ and $\{x, y\} \subset e\}$, where the color of the edge $e \backslash\{x, y\}$ is by definition the color of the edge $e$ in $\mathcal{H}$. Note that $x, y$ are the first two vertices in a $\delta$-bounded selection of Lemma 2.1 thus $\mathcal{H}^{*}$ is a $(1-\delta)$-complete hypergraph. Moreover, since $x, y \in Y_{r-2} \cap Y_{r-1}, \mathcal{H}^{*}$ is colored with $r-3$ colors (colors $r-1$ and $r-2$ cannot be used). Thus, since $r-2 \geq 2$ - induction applies, $\mathcal{H}^{*}$ has an almost perfect monochromatic matching $M$ in its shadow graph. Observing that $M$ is a monochromatic matching in the shadow graph of $\mathcal{H}$ as well, the proof is finished.

\section{References}

1. Berge, C.: Graphs and Hypergraphs. American Elsevier Pub. Co., New York (1973)

2. Figaj, A., Łuczak, T.: The Ramsey number for a triple of long even cycles. J. Combin. Theory Ser. B 97(4), 584-596 (2007)

3. Gyárfás, A., Lehel, J., Sárközy, G.N., Schelp, R.H.: Monochromatic Hamiltonian Bergecycles in colored complete uniform hypergraphs. J. Combin. Theory Ser. B 98(2), 342358 (2008)

4. Gyárfás, A., Ruszinkó, M., Sárközy, G.N., Szemerédi, E.: Three-color Ramsey numbers for paths. Combinatorica 27(1), 35-69 (2007)

5. Gyárfás, A., Sárközy, G.N.: The 3-color Ramsey number of a 3-uniform Berge-cycle. Combin. Probab. Comput. (to appear)

6. Haxell, P., Łuczak, T., Peng, Y., Rödl, V., Ruciński, A., Simonovits, M., Skokan, J.: The Ramsey number for hypergraph cycles I. J. Combin. Theory Ser. A 113(1), 67-83 (2006)

7. Haxell, P., Łuczak, T., Peng, Y., Rödl, V., Ruciński, A., Skokan, J.: The Ramsey number for 3-uniform tight hypergraph cycles. Combin. Probab. Comput. 18(1-2), 165-203 (2009)

8. Łuczak, T.: $R\left(C_{n}, C_{n}, C_{n}\right) \leq(4+o(1)) n$. J. Combin. Theory Ser. B 75(2), 174-187 (1999) 